\title{
O perfil dos estudantes de Odontologia é compatível com o mercado de trabalho no serviço público de saúde brasileiro?
}

Maristela Honório Cayetano*; Mariana Gabriel**; Juliane Tavares***; Maria Ercilia Araújo****; Julie Silva Martins ${ }^{* * * * * ;}$; Edgard Michel-Crosato******; Fernanda Campos de Almeida Carrer********

* Doutoranda, Departamento de Odontologia Social, Faculdade de Odontologia da Universidade de São Paulo. Professora, Universidade de Mogi das Cruzes

** Pesquisadora, Departamento de Odontologia Social, Faculdade de Odontologia da Universidade de São Paulo. Professora, Universidade de Mogi das Cruzes

*** Graduanda, bolsista PIBIC, Faculdade de Odontologia da Universidade de São Paulo

**** Professora Titular, Departamento de Odontologia Social, Faculdade de Odontologia da Universidade de São Paulo

***** Pesquisadora, Departamento de Odontologia Social, Faculdade de Odontologia da Universidade de São Paulo

****** Professor Livre Docente, Departamento de Odontologia Social Faculdade de Odontologia da Universidade de São Paulo

******* Professora Doutora, Departamento de Odontologia Social, Faculdade de Odontologia da Universidade de São Paulo. Coordenadora do Observatório Ibero-americano de Políticas Públicas em Saúde Bucal

Recebido em 19/07/2018. Aprovado em 21/06/2019.

\begin{abstract}
RESUMO
Pesquisadores ao redor do mundo estudam as expectativas, em curto e longo prazo, dos estudantes de último ano de graduação, a fim de buscar evidências científicas que auxiliem gestores e formuladores de políticas públicas na tomada de decisão e no planejamento de suas ações no que diz respeito aos recursos humanos em saúde. O objetivo do presente estudo foi determinar o perfil demográfico e socioeconômico dos estudantes do último ano do curso de Odontologia de uma universidade pública brasileira e relacionar suas perspectivas profissionais com características pessoais, familiares e histórico escolar. Foi realizado um estudo transversal e um questionário foi aplicado aos estudantes do último semestre nos anos de 2014, 2015 e 2016 na Faculdade de Odontologia da Universidade de São Paulo. Foi realizada a análise descritiva e o teste qui-quadrado foi utilizado para examinar a associação entre a intenção de prestar concurso público e migrar para o interior do país e as outras variáveis. A taxa de resposta foi de $82 \%$. Dentre os estudantes da amostra, $76,52 \%$ moravam com os pais. A maioria (82\%) cursou escola privada e somente 7,95\% ingressou no curso por programas de quotas. Quanto às expectativas em relação ao futuro profissional, $70 \%$ pretendem trabalhar como autônomo, 61,36\% no serviço público e somente $28,7 \%$ pretendem trabalhar no ensino. Este foi um estudo exploratório, com os limites descritos na literatura sobre a abordagem metodológica. Entretanto, pesquisas futuras devem avançar nas análises e incorporar metodologias qualitativas a fim de compreender em profundidade fenômenos tais como expectativa profissional, relação do aluno com o Sistema Único de Saúde e mercado de trabalho.

Descritores: Recursos Humanos em Saúde. Cirurgiões-Dentistas. Sistema Único de Saúde.
\end{abstract}




\section{INTRODUÇÃO}

O Fórum Mundial de Recursos Humanos em Saúde 1 (RHS) declarou que "não existe sistema de saúde sem recursos humanos", portanto o planejamento e as políticas públicas em saúde devem considerar as características pessoais e expectativas dos estudantes quanto ao ingresso no mercado de trabalho, a fim de garantir uma tomada de decisão sustentada por evidência científicas, no sentido de regular os estoques de RHS de um país.

O Sistema Único de Saúde (SUS) brasileiro é um sistema de saúde universal, financiado por impostos, que atente mais de 200 milhões de habitantes, em um cenário de grandes desigualdades locorregionais. No Brasil, o profissional pode escolher seu local de atuação e se vai prestar serviço ao sistema público ou privado, lembrando que a prática mista (pública e privada) é uma realidade em todo o território nacional, resultando em conflitos de interesses que podem comprometer a qualidade do serviço prestado no SUS $^{2,3}$. Com a criação do SUS, surgiram as discussões sobre os currículos das profissões de saúde e como adequá-los a fim de formar profissionais capazes de atuar neste sistema de forma integral e equânime ${ }^{4}$.

Em 2004, foi criada a Política Nacional de Saúde Bucal, denominada de Brasil Sorridente, que resultou no aumento da contratação de cirurgiõesdentistas no sistema público. Em 10 anos, só na atenção básica, esse número cresceu mais de $60 \%{ }^{5}$, colocando o SUS como uma possibilidade concreta de atuação profissional para o cirurgião-dentista brasileiro. Pesquisadores ao redor do mundo estudam as expectativas, em curto e longo prazo, dos estudantes de último ano de graduação, a fim de buscar evidências científicas que auxiliem gestores e formuladores de políticas públicas na tomada de decisão e no planejamento de suas ações no que diz respeito aos $\mathrm{RHS}^{6}$. Na Odontologia em particular, destacam-se os estudos de Gallagher et al. (2009) ${ }^{7}$, Baharvand et al. $(2011)^{8}$, Rashid et al.
$(2013)^{9}$, entretanto poucos estudos brasileiros sobre o tema relacionam a questão dos recémformados com essa nova abordagem do mercado, com foco no sistema público de saúde ${ }^{10-12}$.

Nesse contexto o objetivo do presente estudo é determinar o perfil demográfico e socioeconômico dos estudantes do último ano do curso de Odontologia em uma universidade pública brasileira e relacionar suas perspectivas profissionais com características pessoais, familiares e histórico escolar, possibilitando assim discussões que colaborem com diagnóstico situacional e com a construção de evidências no processo de informação em Odontologia.

\section{METODOLOGIA}

Foi realizado um estudo transversal para avaliar três dimensões relacionadas aos estudantes: i) características pessoais; ii) trajetória educacional; iii) escolhas profissionais a curto e longo prazos. Em algumas questões, os estudantes poderiam assinalar mais de uma alternativa.

O questionário foi aplicado aos estudantes do último semestre na disciplina de Gestão e Planejamento em Odontologia nos anos de 2014, 2015 e 2016 na Faculdade de Odontologia da Universidade de São Paulo (FOUSP). A Universidade de São Paulo (USP) localiza-se na zona urbana na maior instituição de ensino superior e pesquisa no Brasil. É uma instituição pública e gratuita em que os estudantes são selecionados por um exame de admissão.

Foi utilizado questionário elaborado por um grupo de pesquisadores do Observatório de Recursos Humanos em Odontologia (OPAS/Ministério da Saúde do Brasil), em 2013, a fim de contemplar as características específicas dos estudantes brasileiros. Este instrumento foi testado e ajustado antes da pesquisa com base nas informações e sugestões dos participantes e pesquisadores que realizaram esses testes (piloto), assim como nos grupos focais ${ }^{13}$ e entrevistas 
conduzidas pelo último autor desse artigo, que direcionou sua elaboração.

As análises descritiva e univariada demonstraram os achados do estudo e as características da amostra. $\mathrm{O}$ teste qui-quadrado foi utilizado para examinar a associação entre a intenção de prestar concurso público e migrar para o interior do país e as diferentes variáveis estudadas na pesquisa. Para todos os testes, a significância adotada foi $\mathrm{p}$ menor que 0,05 (5\% de nível de significância), e todas as análises foram obtidas utilizando o SPSS versão 20.0 (Chicago, IL, USA). Essa pesquisa foi aprovada no Comitê de ética e Pesquisa da Universidade de São Paulo sob $n^{\circ}$ 803.834 (CAAE: 35560314.1.0000.0075).

\section{RESULTADOS}

Os achados desse estudo serão apresentados conforme as categorias de análise adotadas, ou seja, i) características pessoais dos estudantes, ii) trajetória educacional e, por fim, iii) expectativas em relação ao futuro profissional.

\section{i) Dados Pessoais dos estudantes}

A taxa de resposta ao questionário foi de 82\% e a amostra foi composta por 264 voluntários, sendo $68 \%(n=179)$ deles do sexo feminino e $32 \%$ $(\mathrm{n}=85)$ do sexo masculino, com média de idade de 24,4 anos. No último ano da graduação $76,52 \%$ $(\mathrm{n}=202)$ morava com os pais/responsáveis e em $90,9 \%$ sua moradia era financiada por pais/responsáveis $(n=240)$, independente de residirem ou não com esses.

Quanto ao perfil socioeconômico, 44,31\% $(\mathrm{n}=117)$ dos pais dos alunos possuíam Ensino Superior e 32,5\% [n=86] Pós-graduação. Já em relação aos estudos anteriores, 82,95\% $(n=219)$ estudaram em escola privada em maior parte do ensino fundamental e $82,58 \%(\mathrm{n}=218)$ no ensino médio. Somente $7,95 \%(\mathrm{n}=21)$ deles ingressaram na faculdade por programas de inclusão (tabela1).

\section{ii) Trajetória educacional}

Durante a graduação, $84,8 \%(n=224)$ dos estudantes tiveram o material odontológico, necessário para cursar as disciplinas clínicas, custeado pelos pais/responsáveis. Dos respondentes, 41,28\% $(n=109)$ acreditam que somente a graduação traria possibilidades adequadas para a inserção no mercado de trabalho (tabela 2).

\section{iii) Expectativas em relação ao futuro profissional}

Na tabela 3, pode-se observar que a grande maioria dos estudantes $(98,4 \% \mathrm{n}=260)$ relata que pretende atuar na área de Odontologia, sendo que, $70 \%(\mathrm{n}=185)$ afirma que deseja exercer a profissão em consultório próprio, 61,36\% (n=162) em instituição pública, 43,9\% (n=116) em empresa privada, $28,7 \%$ [n=76] em área de ensino e $58,71 \%$ $[\mathrm{n}=155]$ pretende atender em convênio ou cooperativa. Quanto à expectativa de renda, $34,46 \%$ ( $n=91$ ) esperam receber após 1 ano de formado, entre 5 e 7 Salários Mínimos e 41,66\% $(\mathrm{n}=110)$ após 5 anos de formado, entre 10 e 20 Salários Mínimos.

Em relação aos cursos de pós-graduação, $74,24 \%(\mathrm{n}=196)$ pretendem realizar atualização ou cursos de curta duração, 60,6\% (n=160) especialização, $31,43 \%(n=83)$ cursos com até 7 dias de duração, $31 \%(\mathrm{n}=82)$ mestrado, $7,57 \%$ $(n=20)$ doutorado e 2,65\% (n=7) pós-doutorado.

Entre os estudantes analisados, 76,51\% $(\mathrm{n}=202)$ pretendem prestar concurso público na área da odontologia e quando questionados sobre a possibilidade de trabalharem em regiões rurais, cidades pequenas ou periferias de grandes cidades $60,98 \%(n=161)$ estariam dispostos a migrar para essas regiões. Aqueles que assinalaram as opções não ou não sei $(38,25 \%$; $\mathrm{n}=101$ ), não iriam por considerar que nessas regiões as condições de trabalho não são adequadas $(44,55 \% ; n=45)$, possuírem laços 
Tabela 1. Características da amostra

\begin{tabular}{|c|c|c|}
\hline Variável & $\mathbf{n}$ & $\%$ \\
\hline \multicolumn{3}{|l|}{ 1. Sexo: } \\
\hline a. Masculino & 85 & 32,00 \\
\hline b. Feminino & 179 & 68,00 \\
\hline \multicolumn{3}{|l|}{ 2. Nasceu em: } \\
\hline a. São Paulo/Grande São Paulo & 230 & 87,12 \\
\hline b. Outro & 34 & 12,88 \\
\hline \multicolumn{3}{|l|}{ 3. Atualmente mora: } \\
\hline a. São Paulo/Grande São Paulo & 259 & 98,11 \\
\hline b. Outro & 5 & 1,89 \\
\hline \multicolumn{3}{|l|}{ 4. Residia com os pais: } \\
\hline a. Sim & 202 & 76,52 \\
\hline b. Não & 62 & 23,48 \\
\hline \multicolumn{3}{|l|}{ 5. Moradia financiada pelos pais: } \\
\hline a. Sim & 240 & 90,09 \\
\hline b. Não & 24 & 9,08 \\
\hline \multicolumn{3}{|l|}{ 6. Maior grau de estudo dos pais: } \\
\hline a. Não possui estudo & 1 & 0,37 \\
\hline b. Ensino Fundamental Completo & 8 & 3,03 \\
\hline c. Ensino Fundamental Incompleto & 11 & 4,07 \\
\hline d. Ensino Médio Completo & 24 & 9,09 \\
\hline e. Ensino Médio Incompleto & 2 & 0,75 \\
\hline f. Técnico Completo & 13 & 4,92 \\
\hline g. Técnico Incompleto & 2 & 0,75 \\
\hline h. Ensino superior completo & 117 & 44,31 \\
\hline i. Pós-Graduação & 86 & 32,50 \\
\hline j. Desconheço essa informação & - & - \\
\hline \multicolumn{3}{|l|}{ 7. Estudou o ensino fundamental em: } \\
\hline a. Escola Privada & 219 & 82,95 \\
\hline b. Escola Pública & 44 & 16,67 \\
\hline c. Não responderam & 1 & 0,38 \\
\hline \multicolumn{3}{|l|}{ 8. Estudou o ensino médio em: } \\
\hline a. Escola Privada & 218 & 82,58 \\
\hline b. Escola Pública & 45 & 17,05 \\
\hline c. Não responderam & 1 & 0,38 \\
\hline \multicolumn{3}{|c|}{ 9. Ingressou na faculdade por Programa de inclusão: } \\
\hline a. Sim & 21 & 7,95 \\
\hline b. Não & 242 & 91,67 \\
\hline c. Não responderam & 1 & 0,37 \\
\hline
\end{tabular}

Tabela 2. Trajetória educacional dos estudantes

\begin{tabular}{lcc}
\hline Variável & n & \% \\
\hline 10. Material utilizado durante a faculdade foi custeado por: & & \\
a. Pais /responsáveis & 24 & 84,8 \\
b. Eu mesmo(a) & 25 & 9,46 \\
c. Familiar & 9 & 3,40 \\
d. Cônjuge & 2 & 0,75 \\
e. Outros & 4 & 1,51 \\
11. Considera que somente a graduação possibilitará condições adequadas para \\
Sua inserção no mercado de trabalho: & & \\
a. Sim & 109 & 41,28 \\
b. Não & 142 & 53,70 \\
c. Não se aplica & 12 & 4,54 \\
Não responderam & 1 & 0,37 \\
\hline
\end{tabular}


Tabela 3. Expectativas dos estudantes em relação ao futuro profissional

\begin{tabular}{lcc}
\hline Variável & $\mathbf{n}$ & $\mathbf{\%}$ \\
\hline 12. Pretende atuar na área da Odontologia? & 260 & 98,48 \\
a. Sim & 4 & 1,51 \\
b. Não & & \\
13. Pretende trabalhar: & 185 & 70,00 \\
a. Em consultório/clínica próprio & 162 & 61,36 \\
b. Em instituição pública (Unidade Básica de Saúde, CEO, & \\
Hospitais) & 116 & 43,93 \\
c. Em empresa privada no setor de odontologia & 76 & 28,78 \\
d. Na área de ensino & 7 & 2,65 \\
e. Outros & 3 & 1,13 \\
Não responderam & & \\
14. Pretende atender em algum convênio ou cooperativa? & 155 & 58,71 \\
a. Sim & 91 & 34,46 \\
b. Não & 25 & 9,46 \\
c. Não se aplica & 3 & 1,13 \\
Não responderam & 202 & 76,51 \\
15. Pretende realizar concurso público? & 57 & 21,59 \\
a. Sim & 5 & 1,89 \\
b. Não &
\end{tabular}

16. Expectativa de renda após 1 ano de formado (considerar média mensal):
a. 0 a 2 salários mínimos
$3 \quad 1,13$
b. 2 a 4 salários mínimos
15,90
c. 4 a 5 salários mínimos
27,65
d. 5 a 7 salários mínimos
34,46
e. 7 a 9 salários mínimos
12,87
f. 10 a 20 salários mínimos
6,43
g. Acima de 20 salários mínimos
0,75
h. Não se aplica
0,75

17. Expectativa de renda após 5 anos de formado (considerar média mensal):
a. 0 a 2 salários mínimos
b. 2 a 4 salários mínimos
c. 4 a 5 salários mínimos
d. 5 a 7 salários mínimos
10,22
e. 7 a 9 salários mínimos
24,62
f. 10 a 20 salários mínimos
41,66
g. Acima de 20 salários mínimos
18,56
h. Não se aplica

18. Cursos de pós-graduação que pretende realizar até 1 ano de formado(a):*

a. Cursos com até 7 dias de duração

31,43

b. Especialização

c. Atualização ou outros cursos de curta duração

60,6

d. Mestrado

74,24

e. Doutorado

f. Pós-doutorado

7,57

g. Outros

2,65

5,68

19. Estaria disposto a exercer a odontologia em zonas rurais, cidades pequenas ou periferias das grandes cidades?
a. Sim
b. Não
c. Não sei
17,04
Não responderam

$\begin{array}{ll}45 & 17,04 \\ 56 & 21,21\end{array}$

$2 \quad 0,75$

(continua) 
(continuação)

20. Caso tenha respondido Não/Não sei para pergunta anterior, o que te impediria:

$\begin{array}{lcc}\text { a. Condições de trabalho não adequadas } & 45 & 44,55 \\ \text { b. Laços afetivos (como família, namorado, amigos) } & 72 & 71,28 \\ \text { c. Acredita que em grandes cidades o salário seja maior } & 14 & 13,86 \\ \text { d. Acostumado à infraestrutura de uma cidade grande } & 48 & 47,52 \\ \text { e. Acredita que a qualidade de vida nas grandes cidades seja } & 11 & 10,89 \\ \text { melhor } & & \\ \text { f. É nascido em uma grande cidade e não tem motivos que o } & 33 & 32,67 \\ \text { leve a se mudar } & & \\ \text { g. Estabilidade em um emprego } & 19 & 18,81 \\ \text { h. Nunca pensei no assunto } & 16 & 15,84 \\ \text { i. Outros } & 3 & 2,97 \\ \text { Não responderam } & 2 & 1,98\end{array}$

21. E independente da sua reposta na questão $n^{\circ} 19$, por qual renda você estaria disposto?

$\begin{array}{lcc}\text { a. } 0 \text { e } 2 \text { SM } & - & - \\ \text { b. } 2 \text { e } 4 \text { SM } & 7 & 2,65 \\ \text { c. } 4 \text { e } 5 \text { SM } & 17 & 6,43 \\ \text { d. } 5 \text { e } 7 \text { SM } & 40 & 15,15 \\ \text { e. } 7 \text { e } 9 \text { SM } & 52 & 19,69 \\ \text { f. } 10 \text { e } 20 \text { SM } & 67 & 25,37 \\ \text { g. Acima de 20 SM } & 53 & 20,07 \\ \text { h. Nenhuma } & 16 & 6,06 \\ \text { i. Não se aplica } & 7 & 2,65 \\ \text { Não responderam } & 3 & 1,13\end{array}$

afetivos $(71,28 \% ; \mathrm{n}=72)$, acreditarem que em grandes cidades o salário seja maior $(13,86 \%$; $\mathrm{n}=14$ ), estarem acostumados à infraestrutura de uma cidade grande $(47,52 \% ; \mathrm{n}=48)$, acreditarem que qualidade de vida nas grandes cidades seja melhor $(10,89 \% ; n=11)$, ter nascido em uma grande cidade e não ter motivos para mudar-se $(32,67 \%$; $\mathrm{n}=33)$, ter estabilidade em um emprego $(18,81 \%$; $\mathrm{n}=19)$, nunca ter pensado sobre $\mathrm{o}$ assunto $(15,84 \%$; $\mathrm{n}=16$ ).

Independente da resposta para essa questão, $25,37 \%$ deles $(\mathrm{n}=67)$ estariam dispostos a atuar nessas regiões por uma renda entre 10 e 20 salários mínimos.

Os testes mostraram diferenças estatisticamente significantes entre a amostra de voluntários que estariam dispostos a prestar concurso público e aquela de estudantes que estariam dispostos a trabalhar em áreas rurais, pequenas cidades e periferias de grandes cidades $(\mathrm{p}<0,05)$. O sexo masculino mostrou-se no limiar de significância para a mesma variável de desfecho $(\mathrm{p}=0,06)$. Quando a variável de desfecho foi trabalhar em áreas rurais, observou-se que as variáveis sexo e prestar concurso foram estatisticamente significantes $(\mathrm{p}=<0,05)$ (tabelas 4 e 5).

\section{DISCUSSÃO}

A taxa de resposta dessa pesquisa foi alta em relação à literatura existente sobre perfil e percepções de estudantes e profissionais da saúde $^{14,15}$, o que fortalece as análises realizadas. $\mathrm{O}$ fato de o questionário ser aplicado dentro de uma disciplina de gestão fez com que os estudantes pudessem refletir sobre o mundo do trabalho e o planejamento da carreira, uma vez que essa fase de transição é desafiadora e a universidade tem o papel de orientar, promovendo momentos curriculares que deem conta dessa importante demanda.

O estudante de graduação é considerado uma importante força de trabalho no sistema de saúde, 
Tabela 4. Distribuição do número e proporção (\%) da amostra segundo pretensão de realizar concurso público após a graduação

\begin{tabular}{|c|c|c|c|c|c|c|c|}
\hline \multirow{2}{*}{ Variável } & \multirow{2}{*}{ Categorias } & \multicolumn{2}{|c|}{ Sim } & \multicolumn{2}{|c|}{ Não } & \multirow{2}{*}{ Total } & \multirow{2}{*}{$p$ do $\chi^{2}$} \\
\hline & & $\mathrm{n}$ & $\%$ & $\mathrm{n}$ & $\%$ & & \\
\hline \multirow[t]{2}{*}{ Sexo } & Feminino & 179 & 67,80 & 85 & 32,20 & 100,0 & 0,06 \\
\hline & Masculino & 85 & 32,20 & 179 & 67,80 & 100,0 & \\
\hline \multirow[t]{2}{*}{ Nasceu em } & São Paulo/Grande São Paulo & 230 & 87,12 & 34 & 12,88 & 100,0 & 0,53 \\
\hline & Outro & 34 & 12,88 & 230 & 87,12 & 100,0 & \\
\hline \multirow[t]{2}{*}{ Mora em } & São Paulo/Grande São Paulo & 259 & 98,11 & 5 & 1,89 & 100,0 & 0,88 \\
\hline & Outro & 5 & 1,89 & 259 & 98,11 & 100,0 & \\
\hline \multicolumn{2}{|c|}{ Reside com pais/responsáveis } & 202 & 76,52 & 62 & 23,49 & 100,0 & 0,82 \\
\hline \multirow[t]{3}{*}{ Ensino fundamental } & Escola privada & 219 & 82,95 & 45 & 17 & 100,0 & 0,49 \\
\hline & Escola pública & 44 & 16,67 & 220 & 83,33 & 100,0 & \\
\hline & Não responderam & 1 & 0,38 & 263 & 99,62 & 100,0 & \\
\hline \multirow[t]{3}{*}{ Ensino médio } & Escola privada & 218 & 82,58 & 45 & 17 & 100,0 & 0,44 \\
\hline & Escola pública & 45 & 17 & 219 & 82,96 & 100,0 & \\
\hline & Não responderam & 1 & 0,38 & 263 & 99,58 & 100,0 & \\
\hline \multicolumn{2}{|c|}{ Ingressou por programa de inclusão } & 21 & 7,95 & 242 & 91,67 & 100,0 & 0,84 \\
\hline \multicolumn{2}{|c|}{$\begin{array}{l}\text { Disposto a trabalhar em zonas rurais, cidades } \\
\text { pequenas ou periferias das grandes cidades }\end{array}$} & 161 & 60,98 & 103 & 39 & 100,0 & 0,00 \\
\hline
\end{tabular}

Tabela 5. Distribuição do número e proporção (\%) da amostra que aceitaria trabalhar em zona rural, cidades pequenas ou periferias de grandes cidades

\begin{tabular}{|c|c|c|c|c|c|c|c|}
\hline \multirow{2}{*}{ Variável } & \multirow{2}{*}{ Categorias } & \multicolumn{2}{|c|}{ Sim } & \multicolumn{2}{|c|}{ Não } & \multirow{2}{*}{ Total } & \multirow{2}{*}{$\begin{array}{c}\text { p do } \\
\chi^{2}\end{array}$} \\
\hline & & $\mathrm{n}$ & $\%$ & $\mathrm{n}$ & $\%$ & & \\
\hline \multirow[t]{2}{*}{ Sexo } & Feminino & 179 & 67,8 & 85 & 32,2 & 100,0 & 0,05 \\
\hline & Masculino & 85 & 32,2 & 179 & 67,8 & 100,0 & \\
\hline \multirow[t]{2}{*}{ Nasceu em } & São Paulo/Grande São Paulo & 230 & 87,12 & 34 & 12,88 & 100,0 & 0,41 \\
\hline & Outro & 34 & 12,88 & 230 & 87,12 & 100,0 & \\
\hline \multirow[t]{2}{*}{ Mora em } & São Paulo/Grande São Paulo & 259 & 98,11 & 5 & 1,89 & 100,0 & 0,17 \\
\hline & Outro & 5 & 1,89 & 259 & 98,11 & 100,0 & \\
\hline \multicolumn{2}{|c|}{ Reside com pais/responsáveis } & 202 & 76,52 & 62 & 23,49 & 100,0 & 0,94 \\
\hline \multirow[t]{3}{*}{ Ensino fundamental } & Escola privada & 219 & 82,95 & 45 & 17 & 100,0 & 0,65 \\
\hline & Escola pública & 44 & 16,67 & 220 & 83,33 & 100,0 & \\
\hline & Não responderam & 1 & 0,38 & 263 & 99,62 & 100,0 & \\
\hline \multirow[t]{3}{*}{ Ensino médio em } & Escola privada & 218 & 82,58 & 45 & 17 & 100,0 & 0,87 \\
\hline & Escola pública & 45 & 17 & 219 & 82,96 & 100,0 & \\
\hline & Não responderam & 1 & 0,38 & 263 & 99,58 & 100,0 & \\
\hline \multicolumn{2}{|c|}{ Ingressou por programa de inclusão } & 21 & 7,95 & 242 & 91,67 & 100,0 & 0,85 \\
\hline \multicolumn{2}{|c|}{ Pretende realizar concurso público } & 202 & 76,81 & 57 & 21,67 & 100,0 & 0,00 \\
\hline
\end{tabular}

sendo assim o planejamento da carreira é uma discussão que precisa ser ampliada de forma transversal no ensino, no sentido de preparar o egresso para o mercado e para as necessidades dos sistemas de saúde vigentes ${ }^{16}$.

Observou-se que o perfil do estudante é majoritariamente oriundo de escolas privadas, do sexo feminino, dependente economicamente dos pais, que por sua vez apresentam escolaridade de nível superior, e que os estudantes, na sua maioria, são oriundos de escolas privadas. Embora seja uma realidade imposta no Brasil, o sistema de quotas vem sendo ampliado e precisa ser discutido na perspectiva da democratização do acesso aos cursos de Odontologia para estudantes de baixa renda, já que em comum a este estudo, outros 
pesquisadores demonstraram a elitização do curso de Odontologia no Brasil, principalmente nas universidades públicas ${ }^{17,18}$. Estudos anteriores apontam a importância da diversidade cultural e social dos futuros profissionais da saúde para corrigir as desigualdades de acesso em saúde, pois é reconhecido que estudantes com menor poder aquisitivo são mais propensos a trabalhar com populações com maior venerabilidade social ${ }^{19,20}$.

De acordo com o Ministério da Educação ${ }^{21}$ no Brasil atualmente existem 378 cursos de odontologia ativos, sendo $47 \mathrm{em}$ instituições públicas e 331 em instituições privadas e além disso um grande crescimento em relação aos cursos de pós-graduação. Os estudantes brasileiros demostraram expectativa de realizar uma pósgraduação bem como de um ganho mensal alto com a Odontologia. $\mathrm{Na}$ Índia, $54,4 \%$ dos estudantes de graduação das faculdades privadas da capital demonstraram interesse em realizar curso de especialização após a graduação e $26,6 \%$ em trabalhar no setor privado; quando citado o setor público, 33,5\% demonstraram interesse, porém $58,2 \%$ mostraram neutralidade ${ }^{22}$. Em relação à renda, alguns estudos mostram que a estabilidade financeira é um fator frequente dentre as expectativas quanto à carreira odontológica ${ }^{7,9,23}$. Esse imaginário do estudante de Odontologia, idealizando um profissional especializado e com renda acima da média nacional, demostra que as universidades apresentam importantes lacunas a serem trabalhadas com os estudantes em relação à realidade do mercado de trabalho e as necessidades da população. É preciso uma mudança de paradigma para a valorização da saúde da família, principalmente voltado a atuação na Atenção Primária à Saúde com perfil e competências necessárias para dar conta das demandas em saúde da população $0^{24}$.

Essas questões e divergências referentes ao mercado de trabalho são pertinentes devido à fase pela qual a Odontologia vem passando no Brasil, ou seja, enfrenta-se um momento no qual o serviço público cresceu fortemente, mas os recémgraduados ainda apresentaram desejos e expectativas de atuação em consultório/clínica privada $^{25}$. No presente estudo os dados mostram que a maioria dos estudantes também pretende prestar concurso público. Diante disso e das disparidades em saúde no Brasil, estudiosos apontam a necessidade de políticas de regulação que façam uma sinergia entre a graduação e o mercado de trabalho, destacando que essas duas vertentes não podem ser planejadas de forma isolada, independente da natureza jurídica das instituições. Sejam elas públicas ou privadas, o foco deve ser a relevância das ações realizadas e a adequação a necessidade social ${ }^{26}$.

Certamente os resultados dessa pesquisa permitem uma reflexão sobre os conflitos existentes entre a prática mista e a necessidade de que novas pesquisas avancem na compreensão desse fenômeno identificado no estudo, pois ainda existem muitas lacunas a serem explicadas, como por exemplo, o rendimento financeiro do cirurgiãodentista no serviço público e no serviço privado. A dupla prática é aceita e praticada em diversos países, inclusive no Brasil, no entanto existem importantes evidências que sugerem uma possível redução da acessibilidade e qualidade para os usuários do sistema público de saúde. Um aspecto interessante e que merece destaque é o fato de que nossos estudantes demonstram desejo de se especializar e se atualizar após a formação, e ao mesmo tempo ingressar no setor público, perfil que pode qualificar o serviço e garantir o cuidado necessário ao usuário. Entretanto, o SUS deve estar preparado para receber este profissional motivado e disposto a se capacitar de modo que este aspecto não se perca ao longo dos anos. Políticas de educação permanente devem acolher esta demanda dos recursos humanos em saúde, não perdendo o foco na formação de um profisssional generalista, como indicam as Diretrizes Curriculares Nacionais 
e o próprio SUS. Mais uma vez, destaca-se a importância da universidade no direcionamento da carreira profissional dos estudantes, para que a formação resulte em um profissional que reconheça a realidade e que deseje trabalhar no SUS a fim de contribuir para seu fortalecimento e crescimento $^{21}$ e não deseje ingressar no serviço público apenas no começo de carreira, enquanto se qualifica, para depois atuar no setor privado como especialista.

Os problemas de desequilíbrios regionais de profissionais e a falta de acesso a Odontologia no setor público, somado com os resultados desse estudo, demostram que a maioria dos estudantes estariam dispostos a exercer a Odontologia em zonas rurais, cidades pequenas ou periferias das grandes cidades, apontam que há uma necessidade de melhorar a gestão desse grande contingente de profissionais pelos órgãos competentes ${ }^{27}$. A aproximação da academia com a gestão, no sentido de regular esses eixos e diminuir as disparidades poderia contribuir no sentido de solucionar os problemas do mercado de trabalho e da saúde pública, resultando também em profissionais mais satisfeitos, motivados e engajados com os princípios do SUS. Considerar essa janela de oportunidade para mudar a realidade do país é urgente, reduzir as disparidades de acesso, a partir da oferta de concursos públicos onde existe carência de profissionais, é uma possibilidade real e viável para o país, assim como outras opções que já foram identificadas na literatura, mas ainda não foram implementadas pelos formuladores de política em saúde bucal ${ }^{28}$.

Este foi um estudo exploratório, com os limites descritos na literatura sobre a abordagem metodológica, entretanto, pesquisas futuras devem avançar nas análises e incorporar metodologias qualitativas a fim de compreender em profundidade fenômenos tais como expectativa profissional, relação do estudante com o SUS e mercado de trabalho.

\section{CONCLUSÃO}

$\mathrm{O}$ perfil do egresso a Faculdade de Odontologia da FOUSP apresenta-se como majoritariamente do sexo feminino, jovem (idade média de 24 anos), oriundo de escola privada e dependente financeiramente dos pais/ responsáveis, que por sua vez apresentam ensino superior completo. Em relação ao futuro profissional, a maioria pretende trabalhar em consultório próprio, mas também pretende prestar concurso público. Entre os estudantes avaliados, 60,98\% aceitariam trabalhar em regiões rurais do país, e os $39,02 \%$ que não aceitariam apontaram fatores ligados a piores condições de trabalho e ao estilo de vida na área rural como justificativa. No entanto, $25,37 \%$ dos estudantes estariam dispostos a atuar nessas regiões por uma renda entre 10 e 20 salários mínimos. Na amostra, os egressos do sexo masculino e que almejam prestar concurso se apresentaram mais propensos a atuar na área rural. As universidades devem levar em consideração as expectativas dos egressos e mudanças no mercado de trabalho para preparar profissionais que possam atuar no SUS auxiliando na diminuição das inequidades no acesso aos serviços.

\section{ABSTRACT \\ Is the profile of Dental students compatible with the Brazilian public health service?}

Researchers around the world study the expectations, in the short and long term, of students attending the last year of undergraduate studies in order to seek scientific evidence to assist managers and policy makers in public health service. The objective of this study is to determine the demographic and socioeconomic profile of the students of the last year of a Brazilian public Dentistry university and to relate their professional perspectives with personal, family characteristics. A cross-sectional study was carried out and a questionnaire was applied to students from the last semester of 2014, 2015 
and 2016, at University of São Paulo. The response rate was $82 \%$, and $76.52 \%$ of the students lived with their parents. Most of them $(82 \%)$ studied in private schools in the elementary and high school education and only $7.95 \%$ entered into university being benefited by the quota programs. Regarding expectations concerning the professional future, $70 \%$ intend to work as self-employed professionals, $61.36 \%$ in the public service and only $28.7 \%$ intend to work in education. This was an exploratory study, with the limits described in the literature on the methodological approach. However, future researches should incorporate qualitative methodologies in order to deeply understand phenomena such as the professional expectation, the student relation with the Brazilian Unified Health System (SUS) and the labor market.

Descriptors: Human Resources for Health. Dental Surgeons. Brazilian Unified Health System.

\section{REFERÊNCIAS}

1. Campbell J, Dussault G, Buchan J, PozoMartin F, Guerra Arias M, Leone C, Siyam A, Cometto G. A universal truth: no health without a workforce. Forum Report, Third Global Forum on Human Resources for Health, Recife, Brazil. Geneva, Global Health Workforce Alliance and World Health Organization, 2013.

2. Girardi SN, Carvalho CL, Girardi Júnior JB, Araújo JF. "Configurações do mercado de trabalho dos assalariados em saúde no Brasil." Formação 6. 2002:15-36.

3. Girardi, SN, Fernandes Jr H, Carvalho, CL. A regulamentação das profissões de saúde no Brasil. Espaço para Saúde. 2000;2(1).

4. Zilbovicius C, de Araujo ME, Botazzo C, Frias AC, Junqueira SR, Junqueira CR. A paradigm shift in predoctoral dental curricula in Brazil: evaluating the process of change. J Dent Educ. 2011;75(4):557-64.

5. Pucca GA Jr, Gabriel M, de Araujo ME, de Almeida FC. Ten Years of a National Oral
Health Policy in Brazil: innovation, boldness, and numerous challenges. J Dent Res. 2015;94(10):1333-7.

6. Crettenden IF, McCarty MV, Fenech BJ, Heywood T, Taitz MC, Tudman S. How evidence-based workforce planning in Australia is informing policy development in the retention and distribution of the health workforce. Hum Resour Health. 2014;12(1):7.

7. Gallagher JE, Patel R, Wilson NH. The emerging dental workforce: long-term career expectations and influences. A quantitative study of final year dental students' views on their long-term career from one London Dental School. BMC Oral Health. 2009; 9(1):35.

8. Baharvand M, Moghaddam EJ, Pouretemad H, Alavi K. Attitudes of Iranian dental students toward their future careers: an exploratory study. J Dent Educ. 2011;75(11):1489-95.

9. Rashid HH, Ghotane SG, Abufanas SH, Gallagher JE. Short and long-term career plans of final year dental students in the United Arab Emirates. BMC Oral Health. 2013;13(1):39.

10. de Oliveira Costa, BA, Gonçalves, C F, Zanin, L, Flório, FM. Inserção de egressos de Odontologia do Tocantins no mercado de trabalho. Rev ABENO. 2016;16(2), 93-104.

11. Pinheiro, IAG, Noro, LRA. Egressos de Odontologia: o sonho da profissão liberal confrontado com a realidade da saúde bucal. Rev ABENO. 2016;16(1),13-24.

12. dos Santos, BRM, Gonzales, PS, de Almeida Carrer, FC, de Araújo, ME. Perfil e expectativas dos ingressantes da Faculdade de Odontologia da USP: uma visão integrada com as diretrizes curriculares nacionais e o Sistema Único de Saúde. Rev ABENO. 2015;15(1), 28-37.

13. Parker A, Tritter J. Focus group methods and methodology: current practice and recent debate. Int J Res Method Educ. 2001;29(1):23-37.

14. Freire MC, Jordão LM, de Paula Ferreira N, de 
Fatima Nunes M, Queiroz MG, Leles CR. Motivation towards career choice of Brazilian freshman students in a fifteen-year period. $\mathbf{J}$ Dent Educ. 2011;75(1):115-21.

15. Honey J, Lynch CD, Burke FM, Gilmour AS. Ready for practice? A study of confidence levels of final year dental students at Cardiff University and University College Cork. Eur J Dent Educ. 2011;15(2):98-103.

16. Bozorgmehr K, Saint VA, Tinnemann P. The 'global health' education framework: a conceptual guide for monitoring, evaluation and practice. Global Health. 2011;7(1):8.

17. dos Santos BF, Nicolau B, Muller K, Bedos C, Zuanon AC. Brazilian dental students' intentions and motivations towards their professional career. J Dent Educ. 2013; 77(3):337-44.

18. Sinkford JC, Valachovic RW, Harrison SG. Underrepresented minority dental school enrollment: continued vigilance required. J Dent Educ. 2004;68(10):1112-8.

19. Andersen RM, Carreon DC, Friedman JA, Baumeister SE, Afifi AA, Nakazono TT, Davidson PL. What enhances underrepresented minority recruitment to dental schools? J Dent Educ. 2007;71(8):994-1008.

20. Gabriel M, Cayetano MH, Chagas MM, Araújo ME, Dussault, G, Pucca Júnior GAP, Carrer FCA. Mecanismos de ingresso de cirurgiões dentistas no SUS: Uma agenda prioritária para o fortalecimento do Brasil Sorridente. Ciên Saúde Colet. 2018. [Acesso em 17 jun. 2018]. Disponível em http://www.cienciaesaude coletiva.com.br/artigos/mecanismos-deingresso-de-cirurgioes-dentistas-no-sus-umaagenda-prioritaria-para-o-fortalecimento-dobrasil-sorridente/16916? id=16916

21. Brasil. Ministério da Educação. Portal da Transparência. [Acesso em 17 jun. 2018]. Disponível em: http://emec.mec.gov.br
22. Sharma N, Jain K, Kabasi S. Attitude toward Public Health Dentistry as a career among dental students in Odisha: A Cross-sectional study. Dent Res J (Isfahan). 2016;13(6):532-38.

23. Granja, GL, Santos, JTL, Mariz, RC, Araki AT, Vieira S, Nunes JMFF, Fonseca FRA. Perfil dos estudantes de graduação em Odontologia: motivações e expectativas da profissão. Rev ABENO. 2016;16(4), 107-13.

24. Davis EL, Stewart DC, Guelmann M, Wee AG, Beach JL, Crews KM, Callan RS. Serving the public good: challenges of dental education in the twenty-first century. J Dent Educ. 2007;71(8):1009-19.

25. Sousa JED, Maciel LKB, Oliveira CASD, Zocratto KBF. Mercado de trabalho em Odontologia: perspectivas dos estudantes concluintes de faculdades privadas no município de Belo Horizonte, Brasil. Rev ABENO, 2017; 17(1), 74-86.

26. Ceccim RB. A formação e especialização de profissionais de saúde e a necessidade política de enfrentar as desigualdades sociais $\mathrm{e}$ regionais. Rev Bras Educ Med. 2007;31(3):266-77.

27. Brekke KR, Sørgard L. Public versus private health care in a national health service. Health Econ. 2007;16(6):579-601.

28. Gabriel M, Cayetano, MH, Galante, ML, Carrer FC, Dussalt G, Araujo ME. A Global overview of the geographical distribution of dentists: a scoping review. JDR Clin Transl Res. 2018;3(3):229-37.

\section{Correspondência para:}

Fernanda Campos de Almeida Carrer e-mail: fernandacsa@usp.br

Av. Professor Lineu Prestes, 2227

Cidade Universitária

05508-000 São Paulo/SP 